\title{
Multi-criteria optimization of power systems' modes at incompatible constraints
}

\author{
Rashid Sitdikov $^{1 *}$, Olga Radionova ${ }^{1}$ and Margarita Mandalaka ${ }^{2}$ \\ ${ }^{1}$ Tashkent State Technical University named after Islam Karimov, Uzbekistan \\ ${ }^{2}$ Coordination and Dispatch Center of United power system (UPS) in Central Asia, Tashkent
}

\begin{abstract}
The paper presents a mathematical model for solving the problem of operational optimization of electric power systems (EPS) modes when incompatible restrictions appear with using the mathematical apparatus of multi-criteria optimization. Along with the main criterion, three additional criteria are considered, which, in the calculations, make it possible optimally to enter the modes into the acceptable area.
\end{abstract}

\section{Introduction}

Due to the transition of our country to market relations in the energy sector and their further development, the value of optimization calculations has significantly increased. The statement of any optimization problem, including optimization of modes of electric power systems (EPS), includes optimization goals (criteria) and a system of restrictions. The main criterion for the optimality of an EPS is usually the criterion of the minimum cost (usually fuel) during the time period under consideration. When solving optimization problems, other criteria or a combination of them can be used (multi-criteria optimization problem).

\section{Problem statement}

The solution of optimization problems of the EPS management using the appropriate optimization criteria is always carried out within the limits of restrictions. There are a large number of such restrictions, they are different for different tasks; some of them change their values during the period of operational (daily) optimization, appear or disappear [1-7].

Model constraints - mathematical relations that reflect the properties of modeled objects in relation to external (limitation) factors. Usually representing a system of equations and inequalities, they collectively determine the area of acceptable solutions (the acceptable set).

We will point out some frequently encountered restrictions-inequalities in the operational optimization of EPS modes. For example, restrictions on the maximum capacity of generator groups, their composition, on the speed of power changes, on stability (maximum power flows through power transmission lines), on voltages in nodes, on the maximum levels of hydroelectric power reservoirs, on the specified energy production for the periods of limited power facilities, restrictions on fuel and their types, and many others.
Compatibility of the constraint system is a prerequisite for the classical solvability of the model: if this system is incompatible, the allowed set is empty.

In practice, the constraints often include resources of raw materials, capital investment, environmental aspects, possible options for reconstruction and repair of equipment, reliability and safety, needs for finished products, modes, etc.

As a rule, if you relax the constraints of the problem, the performance of its solution will be better than when solving the problem that meets the real conditions. Conversely, if you make the restrictions more stringent and thereby reduce the options available, the solution will usually be worse. In the first case, it will be optimistic, in the second pessimistic. This opens up the possibility of approximate, estimated solutions to some optimization problems: by changing the constraints, you can estimate the range of values within which the solutions to the problem are located.

Mathematically, constraints are divided into constraints in the form of equalities and inequalities. The number of such restrictions is usually large, since the problems of optimizing the modes of modern EPS are characterized by a large dimension.

Inequality constraints can be rigid or non-rigid; the degree of rigidity of each constraint can vary and depends mainly on their type and mode of EPS.

To solve such problems, as a rule, developed optimization methods are used according to one main criterion. All other criteria are classified as restrictions. In cases where the constraints are incompatible, algorithms based, as a rule, on the methods of penalty functions and "barriers" are used. However, scalar algorithms using the above methods are not always effective under strictly defined constraints, their incompatibility and inconsistency, and in most cases this problem is not solved by ordinary scalar methods at all $[1,3]$.

The incompatibility of the specified restrictions, their inconsistency, is revealed only in the process of the calculation itself. This makes it necessary to review the composition of the operating equipment, change the values

\footnotetext{
* Corresponding author: tstu_energy@list.ru
} 
of the specified parameters and restrictions, and perform new calculations. This process can take a long time, which is unacceptable for operational calculations and their correction.

Let's look at the mathematical expressions of restrictions, their features, as well as the possibility of making changes in operational calculations.

When optimizing the EES mode, for a given set of equipment, usually set limits (generator power, power line flows, voltage values in nodes, etc.) are reduced to a system of inequalities of the form [6]:

$$
Z_{\text {imin }} \leq Z_{i}(\boldsymbol{X}) \leq Z_{\text {imax }} \text {; }
$$

where $Z_{\text {imin }}, Z_{\text {imax }}$ are the set minimum and maximum constraints; $\boldsymbol{X}$ is the vector of controlled parameters; $i=$ $(1, n)$ is the index of the set constraint; $n$ is the number of set constraints.

It is necessary to get a solution that gives an answer to the question: what values should be restricted so that their parameters differ least from the calculated initial values, provided that the General optimization problem is solved. To solve this problem, we present it in a multi-criteria setting. The given constraints in the form of inequalities (1) are represented as partial criteria of the form:

$$
F_{i}(\boldsymbol{X})=\left\{\begin{array}{l}
0, \text { if } Z_{\text {imin }}, \leq Z_{i}(\boldsymbol{X}) \leq Z_{\text {imax }} ; \\
C_{i}\left(Z_{\text {imin }},-Z_{i}(\boldsymbol{X})\right), \text { if } Z_{\text {imin }},>Z_{i}(\boldsymbol{X}) ; \\
C_{i}\left(Z_{\text {imax }}-Z_{i}(\boldsymbol{X})\right), \text { if } Z_{i}(X)>Z_{\text {imax }} .
\end{array}\right.
$$

here $C_{i}>0$ are some weight coefficients showing the degree of rigidity of this restriction.

The solution of the problem, including of inequalities system (1), is reduced to solving a multi-criteria optimization problem:

$$
F_{i}(\boldsymbol{X})=\min .
$$

It is obviouslu, that if there are no values among $X \epsilon D x$ for which all $F_{i}(\boldsymbol{X})=0$, then in the system of constraints (1), there are contradictory ones, i.e. they are incompatible and the problem is not solved by ordinary scalar methods. Here $D_{x}$ is the range of $\boldsymbol{X}$ acceptable values.

The use of modern mathematical methods, in particular the multi-criteria optimization apparatus, makes it possible to overcome the problem of inconsistency and incompatibility of the specified constraints [2-6] in the calculation process. Let's look at ways to solve such problems.

\section{The mathematical model of optimization}

Consider a multi-criteria optimization model consisting of several objective functions, a set of coupling equations, and a set of technological and regime constraints [4].

In general, the mathematical model includes:

- model of electrical mode;

- model of optimization.

In its turn, the optimization model consists of an objective function and a large set of technological and regime restrictions, which are divided into hourly and integral ones.

On the model of the electric mode of the EPS. The main purpose of the model electric mode is calculating on the basis of the electrical model of the power system, the linearized coefficients of the distribution of node capacities for electric grid elements, with a view to their subsequent use in defining the limits of the optimization model.
The electric mode model is based on the following simplifying assumptions:

- active resistance to elements of the grid are equal to zero;

- reactive power flows in the network are not considered;

- the linearity of the electrical mode with respect to the active power, which is equivalent to the constancy of the values of the coefficients of distribution of nodal power over the network elements.

The possibility and necessity of applying these assumptions was determined on the basis of:

- preliminary comparative estimates of the error in determining the flow distribution of active power along lines when calculating the full (with a complex representation of line resistances and node capacities) and simplified (taking into account the above assumptions) models of the electrical mode;

- the fact that the electrical model is applied to a fairly simplified, with a certain error, equivalent to the electrical scheme of an electric power plant, in which the predicted values of consumption and loads of non-calculating stations, enlarged power nodes, and power systems will be used as input mode parameters;

- the need to obtain, within a limited period of time, a reliable guaranteed result when calculating hourly values of linearized coefficients of distribution of nodal capacities across the elements of the electric network, regardless of the input conditions for hourly calculations of steady-state electrical modes.

These assumptions allow us to determine the coefficients of node power distribution through the parameters of network elements in the form of the following generalized expression:

$$
C_{i j}^{q}=\frac{K_{T i j}}{X_{T i j}} \cdot \frac{A_{i} \cdot X_{j q}-A_{j} \cdot X_{i q}}{A_{q}},
$$

here: $C^{q}{ }_{i j}$ is the power distribution coefficient of node $q$ for the $i-j$ branch;

$$
A=Y^{-1} \cdot Y_{\kappa 0}
$$

$Y^{-1}$ is the inverse matrix of nodal conductivities of an equivalent electrical circuit of an EPS;

$Y_{K 0}$ - vector-column of links of the reference node;

$$
K_{T i j}=\frac{U_{i}}{U_{j}} ;
$$

$K_{T i j}$ - transformation coefficient of the branch $i-j$;

$U_{i}, U_{j}$ - voltages of nodes $\mathrm{i}, \mathrm{j}$ that limit the transformer;

$X_{T i j}$ - reactance of the transformer branch $i-j$;

$X_{i q}, X_{j q}$ - the reactances of the $i-q$ and $j-q$ branches.

Taking into account the accepted assumption regarding the linearity of the electric mode with respect to the active power, the active power flows over any network element of the electric model can be determined in the optimization model by the following expression:

$$
P_{i j}=\sum_{Q} C_{i j}^{q} \cdot P_{p}
$$

where: $C^{q} i j$ - power distribution coefficient of node $\mathrm{q}$ for the branch $i-j ; P_{p}$ - active power of the node $q$.

The coefficients $C_{i}>0$ in (3) are weight coefficients that take into account the importance of the $i$-th constraint, the sum of which in the EPS node is $1,\left(\sum C_{i}=1\right)$. And, as already mentioned, the solution of the problem is reduced to solving a multi-criteria optimization problem (3). 
From the above, it follows that the incompatibility of constraints is usually the reason why it is impossible to enter the mode into the allowed range for a given set of independent variables. The allowed area is determined by the specified limits, and the independent variables are usually the power of the stations.

\section{About of algorithm's solution}

In the algorithm developed by us, based on minimization (3), the constraint parameters change automatically when their inconsistency is detected, i.e. the optimal parameter changes are embedded directly in the algorithm for solving such a problem.

This can be implemented either by extending the adjustment range of the main independent variables, or by entering additional independent variables. Moreover, as shown below, in addition to the usual optimization criterion for minimizing the cost of generating electric energy $\left(F_{1}\right)$, criteria are introduced $\left(F_{2}, F_{3}, F_{4}\right)$ that minimize the permissible deviations of the specified restrictions.

Thus, the target functions of the multi-criteria optimization model selected (after theoretical and applied research) are represented as 4 minimized components:

a) the total cost per day for the purchase of energy from the calculated (participating in optimization) power plants:

$$
\mathrm{F}_{1}=\sum_{t=1}^{24} \sum_{q} E_{q}^{t} \cdot P_{q}^{t} \rightarrow \operatorname{Min} ;
$$

where $E_{q}^{t}$ is the selling cost of energy at the station $q$ per hour $t$;

$P_{q}^{t}$ - the value of generating station $q$ per hour $t$;

b) the total cost of energy due to deviations from the minimum power limits of generators at calculation stations:

$$
\mathrm{F}_{2}=\sum_{t=1}^{24} \sum_{q} E_{q}^{t} \cdot K_{g \min q}^{t} \rightarrow \text { Min }
$$

where $K_{g \min q}^{t}$ is the value of the deviation $P_{g} \min$ at the station $q$ per hour $t$;

c) the total cost of energy due to deviations from the limits on the maximum power of generators at calculation stations:

$$
\mathrm{F}_{3}=\sum_{t=1}^{24} \sum_{q} E_{q}^{t} \cdot K_{g \max q}^{t} \rightarrow \text { Min } ;
$$

where $K_{\mathrm{g} \text { max q }}^{\mathrm{t}}$ is the value of the deviation $P_{g \max }$ at the station $q$ per hour $t$;

d) total damage per day from under-supply of energy to consumers due to reduced consumption in nodes (introduction of regulatory measures):

$$
\mathrm{F}_{4}=\sum_{t=1}^{24} \sum_{t=1}^{n} E_{n} \cdot K_{\text {comp } n}^{t} \rightarrow \text { Min }
$$

where $E_{n}$ is the cost of damage from power limitation of 1 MWh in node $n$;

$K_{\text {comp } n}^{t}$ - the amount of consumption reduction in node $n$ per hour $t$.

In the described mathematical model and algorithm, the criteria are ranked: the first one is the main one, and the other three are those that contribute to expanding the area of optimal solutions. This division of criteria allows us to formulate the optimization problem as the problem of finding the extreme of the main criterion, provided that the values of the other criteria are at the Optima identified in the calculation process.

Thus, the criteria $\left(F_{1}, F_{3}, F_{4}\right)$ are introduced into the optimization model to solve it as a tool for entering the EPS modes into the acceptable range in cases of detection of incompatible restrictions.

Practically, this means that in case of incompatibility restrictions, the range of available capacities calculation of stations expands in the direction of reducing the minimum available capacity of the station $\left(P_{g}\right.$ min $)$ and increase of maximum limit of available capacity $\left(P_{g} \max \right)$. Reducing the number of units can be achieved by disabling some of the units or generators. Conversely, an increase in The $P_{g} \max$ can be achieved by turning on additional units or generators, i.e. changing the composition of the operating equipment.

In addition, the need to reduce the $P_{g} \min$ at a single station or at several stations may be caused by locking the power of the station by the network factor or by reducing the total consumption of the EPS below the sum of the $P_{g}$ min of all stations. The need to increase the $P_{g} \max$ of stations can be caused either by a general shortage of generating capacity in the EPS as a whole, or by a shortage of capacity in a separate node or power district due to restrictions on power lines.

It is obviously that the process of expanding the limits of available capacity at calculation stations should be regulated. This can be done by providing the decision maker (dispatcher, expert system [7]) with the ability to determine the optimal adjustment range for each calculation station in the direction of decreasing $P_{g}$ min and increasing $P_{g}$ $\max$.

This circumstance is convenient to implement by setting for each station the coefficients of the adjustment ranges $K_{g}$ $\min$ and $K_{g} \max$. An additional adjustment range using these coefficients will be determined for each station as a proportion of the $P_{g} \min$ and $P_{g} \max$ stations, respectively. In a particular case, by setting the $K_{g \text { min } q}^{t}$ and $K_{g}^{t} \max q$ equal to zero, you can prohibit the consideration of these criteria, i.e. you can prohibit the expansion of the adjustment range at individual stations.

As another additional regulated parameter and corresponding minimization criterion $\left(\mathrm{F}_{4}\right)$, the regulated part of node consumption is used. In practice, if there is a lack of generating capacity in the whole of the EPS or in a separate energy district due to restrictions on power lines, as a means of regulation when planning and maintaining the EPS modes, a partial shutdown of the load in the nodes is practiced-the introduction of regulatory measures. This approach is implemented in the optimization model in the form of a task for the nodes of the adjustment range in the direction of changing consumption. The size of the adjustment range, as well as in the case of station restrictions, is determined by the decision maker (DM) through the share coefficient of the $K_{\text {comp } n}^{t}$, which affects the consumption of the node.

The above three complementary criteria that extend the acceptable optimization area are automatically introduced and used in cases of deficient, unbalanced modes and incompatible restrictions, and if the mode is balanced and there are no incompatible restrictions, their values can be minimal or equal to zero. 
Let's consider the coupling equations and constraints corresponding to this model.

The coupling equations represent the relationships between the input and output parameters of the power system elements, such as the consumption characteristics of the TPP $\left(\mathrm{B}_{\mathrm{q}}=\mathrm{f}_{\mathrm{q}}\left(\mathrm{P}_{\mathrm{q}}\right)\right)$, HPP $\left(\mathrm{Q}_{\mathrm{q}}=\left(\psi_{\mathrm{q}}\right)\right)$, the starting characteristics of the TPP $\left(B_{s q}=\varphi_{q}\left(G_{q}\right)\right)$, power losses in the transmitting and converting elements of the network, transformation coefficients, adjustment characteristics of load nodes, etc.

The hourly limits. The limits taken into account during optimization for each hour of the settlement day include:

limits on the available capacity of settlement stations:

$$
\mathrm{P}_{\text {minq }} \leq \mathrm{P}_{\mathrm{q}} \leq \mathrm{P}_{\text {maxq }} \text {; }
$$

limitations on the increment of $P_{\max q}$ :

$$
0 \leq \mathrm{K}_{\mathrm{g} \max } \mathrm{u}_{\mathrm{r}} \mathrm{g} \max \mathrm{q} \mathrm{P}_{\max \mathrm{q}} ;
$$

where $K_{g} \max q$ is the increment of $P_{\max }$ at station $q$;

$K_{r g \max q}$ - the share coefficient for calculating the additional adjustment range $P_{\max }$ at station $q$;

limitations on the increment of $P_{\min } q$ :

$$
0 \leq \mathrm{K}_{\mathrm{g} \min \mathrm{q}} \leq \mathrm{K}_{\mathrm{r} g \min \mathrm{q}} \mathrm{P}_{\min \mathrm{q}}
$$

where $K_{g} \min q$ is the increment of $P_{\min }$ at station $q$;

$K_{r g \min q}$ - the share coefficient for calculating the additional adjustment range $P_{\min }$ at the station $q$;

$\mathrm{P}_{\text {cons } \mathrm{n}}$ - the specified consumption in node $n$; restrictions on overflows of active power of controlled power lines:

$$
P_{i j \min } \leq \sum_{q} C_{i j}^{q} \cdot\left(P_{q}+K_{g \max q}-K_{g \text { min } q}\right)+\sum_{n} C_{i j}^{n} \cdot K_{c o n s n} \leq P_{i j \max } ;(15
$$

where $C^{q}{ }_{i j}$ - coefficients of power distribution of stations $q$ along the line $i-j(i, j$ - numbers of nodes that define the line in the calculation scheme);

restrictions on active power flows of controlled sections:

$$
P_{\text {seen nin }} \leq \sum_{m} \sum_{q} C_{i j}^{q} \cdot\left(P_{q}+K_{g \max q}-K_{g \text { min } q}\right)+\sum_{n} C_{i j}^{n} \cdot K_{\text {cons }} \leq P_{\text {seen max }} ;
$$

where $m$ is the set of lines $i-j$ included in the section of lines $n$;

restrictions on the balance of the active power of the EPS:

$$
\sum_{q}\left(P_{q}+K_{g \max q}-K_{g \min q}\right)=\sum_{k} P_{f i x k}-\sum_{n} K_{\text {cons } n} ;
$$

where the $P_{f i x}$ is the sum of the set values of consumption, loads of non - calculation stations, external flow and fixed loads of calculation stations in node $k$ of the calculation scheme;

$K_{\text {cons } n}$ - value to reduce the consumption at node $n$.

Integral constraint. They include:

restrictions on the balance of the active capacity of the power system s per day:

$$
\mathrm{C}_{\mathrm{s} \text { giv }}-\mathrm{C}_{\mathrm{s}} \leq\left(\mathrm{C}_{\mathrm{s} \text { giv }} \mathrm{K}_{c}\right) / 100 \text {; }
$$

where $C_{s}$ giv is the set value of the power system $s$ balance per day;

$K_{c}$ - is common to all of the EPS factor accuracy of accounting of limitation by balance ( in $\%$ of $\mathrm{C}_{\mathrm{s} \text { giv }}$ );

the calculated value of the balance flow of the power system $s$ :

$$
C_{s}=\sum_{t=1}^{24}\left(P_{f i x s}^{t}-\sum_{q_{s}^{e}}\left(P_{q}^{t}+K_{g \max q}^{t}-K_{g \min q}^{t}\right)-\sum_{n_{s}^{e}} K_{c o n s n}^{t}\right) ;
$$

$P_{f i x} t_{s}$ - the amount specified in hour $t$ consumption values, loads, not calculated stations, external overflow and fixed load calculated stations of the power system $s$;
$\sum_{q_{s}^{e}}\left(P_{q}^{t}+K_{g \max q}^{t}-K_{g \min q}^{t}\right)$ - total load of calculation stations per hour $t$;

$\sum_{n_{s}^{e}} K_{\text {consn }}^{t}$ - the amount of reduction in consumption per hour $t$ in nodes $n$ corresponding to the power system $s$; restrictions on the total daily energy consumption at the settlement station ' $q$ ':

$$
\mathrm{Q}_{\text {giv q }}-\mathrm{Q}_{\mathrm{q}} \leq\left(\mathrm{Q}_{\text {giv q }} \mathrm{K}_{\mathrm{q}}\right) / 100 \text {; }
$$

where $Q_{g i v}$ is the daily value of energy consumption at the calculation station $q$;

$K_{q}$ - common for all calculation stations coefficient of accuracy of accounting for restrictions on energy consumption (in $\%$ of $\mathrm{Q}_{\text {giv }}$ );

$$
Q_{s}=\sum_{t=1}^{24}\left(P_{q}^{t}+K_{g \max q}^{t}-K_{g \min q}^{t}\right) \cdot R_{q} ;
$$

$R_{q}$ - specific energy consumption at the station $q$;

limitations on total power generation per day at the calculation station $q$ :

$$
\mathrm{W}_{\text {giv q }}-\mathrm{W}_{\mathrm{q}} \leq\left(\mathrm{W}_{\text {giv }} \mathrm{K}_{\mathrm{w}}\right) / 100
$$

where $W_{g i v} q$ is the daily value of power generation at the station ' $q$ ';

$K_{w}$ - the coefficient of accuracy of accounting for the power generation limit common to all calculation stations (in $\%$ of $W_{\text {giv q) }}$.

$$
W_{q}=\sum_{t=1}^{24}\left(P_{q}^{t}+K_{g \max q}^{t}-K_{g \min q}^{t}\right) .
$$

The described mathematical model is implemented in the form of an algorithm and a program for optimizing the daily modes of the United power system (UPS) in Central Asia (CA) [8-10].

The program is in commercial operation in market conditions and provides a solution to the problems of operational optimization of the Central Asian UPS modes:

- hourly optimization of electric power modes of the Central Asian UPS in the billing period;

- minimization of the total costs of the Central Asian UES for the purchase of energy from its producers (main TPP and HPP of the Central Asian UPS);

- accounting for mode and technological restrictions;

- optimal parameter correction in case of incompatibility of restrictions;

- high reliability of the final result.

\section{Conclusion}

In the course of the conducted research and many practical calculations, it is confirmed that multi-criteria optimization of EPS modes makes it possible to effectively solve problems of operational optimization and their correction, including when incompatible or contradictory restrictions appear. The introduction of complementary criteria for optimizing operational planning expands the range of solutions and allows for a high degree of reliability to enter the EPS mode into the acceptable range by optimally changing the ranges of mode and technological restrictions.

The algorithm and program are constantly supplemented and improved: specified the various parameters and ratios, improved control systems and the accuracy of the input data 
(measurements), and expanding the use of elements of digitalization and intellectualization.

\section{References}

1. Fazylov H.F., Nasirov T.H. Steady-state modes of electric power systems and their optimization. Tashkent: Moliya, 1999, $370 \mathrm{p}$.

2. Venikov V.A., Zhuravlev V.G., Filippova T.A. Optimization of power plants and power systems. Moscow: Energoatomizdat, 1990.

3. Arakelyan E.K., Pikina G.A. Optimization and optimal management. Moscow: MEI publishing house, 2003, 356 p.

4. Sitdikov R. A. On multi-criteria of the target function of optimization of EPS modes //Problems of energy and resource saving. 2006, № 3, p. 54-61.

5. Optimization of power system modes. /Under the editorship of V.M. Sinkov. Kiev: Vyshcha SHKOLA, 1976, $308 \mathrm{p}$.
6. Arzamastsev D.A., Bartholomew, P.I., Holyan A.M. ASU and optimization of power system. M.: Higher school,1983, $208 \mathrm{p}$.

7. Nasirov T.Kh., Sitdikov R.A., Udovichenko V.B. From the experience of developing an expert system for managing the modes of electric power systems. /n the collection "Current issues in the field of technical and fundamental Sciences", Issue 2, Tashkent, 2001, pp. 56-62.

8. Sitdikov R.A., Radionova O.V., Mandalaka M.D. Operational optimization of daily modes of the United power system of Central Asian countries //Problems of energy and resource saving, 2007, no. 3-4, p. 47-53.

9. Sitdikov R.A., Radionova O.V., Mandalaka M.D. On solving the problem of optimizing the UPS mode under incompatible restrictions //Problems of energy and resource saving, 2010, no. 1-2, pp. 203-210.

10. Mandalaka M.D., Sitdikov R.A., Mirzaev A.T., Shamsiev H.A. Optimization of electric power systems modes under incompatible restrictions // Problems of energy and resource saving, 2013, №1-2, pp. 58-64. 